\title{
Renaissance der operativen Dermatologie in der Nachkriegszeit. Entwicklung in der DDR
}

\author{
Renaissance of Surgical Dermatology after World War II. Development in the GDR
}

Autor

Institut

\section{A. Scholz}

ehemaliger Leiter des Instituts für Geschichte der Medizin, Medizinische Fakultät Carl Gustav Carus, TU Dresden

\section{Bibliografie}

DOI 10.1055/s-0029-1214786 Online-Publikation: 8. 6. 2009 Akt Dermatol 2009; 35: 275-278 @ Georg Thieme Verlag KG Stuttgart · New York ISSN 0340-2541

Korrespondenzadresse Prof. Dr. (em.) Albrecht Scholz Mendelssohnallee 30 01309 Dresden albrecht.scholz@yahoo.de

\section{Zusammenfassung \\ $\nabla$}

Biografien und Aktivitäten der Gründergeneration der operativen Dermatologie in den 3 Jahrzehnten nach dem Ende des 2. Weltkrieges werden geschildert. Die Vorgeschichte der Gründung einer Sektion „Chirurgische Dermatologie“ in der

Die Medizin der ersten Jahre nach dem Ende des 2. Weltkrieges sah sich in erster Linie durch die seuchenartig auftretenden Krankheiten wie Tuberkulose, Typhus, Fleckfieber und Geschlechtskrankheiten herausgefordert. Dementsprechend war unser Fachgebiet vorrangig mit der Bekämpfung von Syphilis und Gonorrhoe beschäftigt. Der Bereich der operativen Dermatologie konzentrierte sich in wenigen Zentren auf die Behandlung von Kriegsfolgen und auf die operative Behandlung des Lupus vulgaris. Hauttumoren waren in dieser Zeit kaum eine Aufgabe chirurgischer Therapie, da sie vorrangig der Röntgentherapie zugeführt wurden [1].

Zwei Kliniken, die vor und während des Krieges sich mit chirurgischen Interventionen beschäftigt hatten, sollen herausgestellt werden, da sie diese Tradition in die Nachkriegszeit weitergetragen haben: Münster und Düsseldorf.

Carl Moncorps (1896-1952) leitete die Universitäts-Hautklinik Münster von 1938 bis 1952 (๑ Abb. 1).

Er hatte während seiner Assistentenjahre eine Ausbildung in plastischer Chirurgie bei Johannes Esser in Holland und bei Jakob Joseph in Berlin erhalten, die seine gesamte weitere Arbeit bestimmte. Dementsprechend förderte er die Arbeit an der mit der UHK Münster verbundenen Lupusheilstätte Hornheide bei Münster nachhaltig. Bis zum Beginn der Chemotherapie des Lupus vulgaris in den 50er-Jahren, ausgehend von Hornheide, stand hier die operative Therapie im Vordergrund. Folgeschäden von Kriegsverletzungen wa-
DDR wird beschrieben. Von der Hautklinik Dresden unter dem Direktorat von Heinz Egon Kleine-Natrop ausgehend, werden die Gründung 1982, die durchgeführten Arbeitstagungen und die Operationskurse in den Jahren bis zur Wiedervereinigung 1990 vorgestellt.

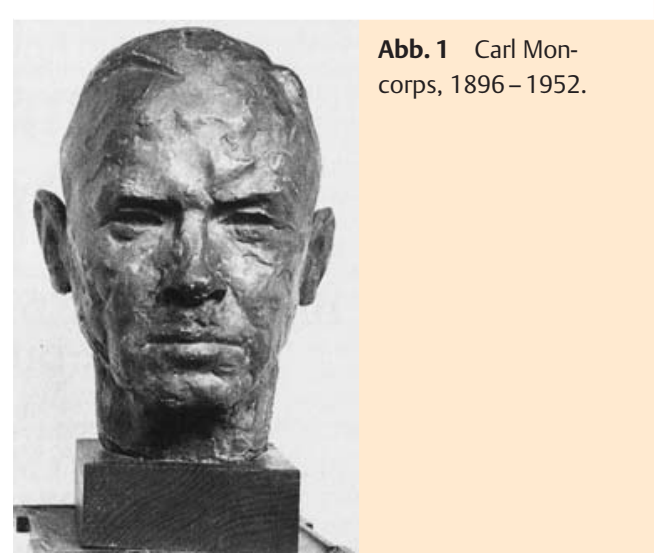

ren die Basis für die spätere in Hornheide großartig realisierte plastische Chirurgie.

Das zweite, die Tradition weiterführende Zentrum war die Universitäts-Hautklinik Düsseldorf, die von 1930 bis 1962 von Hans Theodor Schreus (1892 - 1970) geleitet wurde ( $\bullet$ Abb. 2).

Zwei Innovationen für die Dermatochirurgie sind mit seinem Namen verbunden: Die 1942 von ihm eingeführte, wenn auch durch die Zeitumstände erst 1950 publizierte Chlor-Zink-Schnellätzung und 1949 die mit der Firma Schumann technisch perfektionierte Dermabrasion mit dem hochtourigen Schleifgerät. Aus dem Überblick der vergangenen Jahrzehnte muss der Einsatz von HansTheodor Schreus für die ganzheitliche Betrachtung des hautkranken Menschen mit seinem umfassenden Konzept hervorgehoben werden, das Ärzte verschiedener Fachrichtungen 1955 unter 


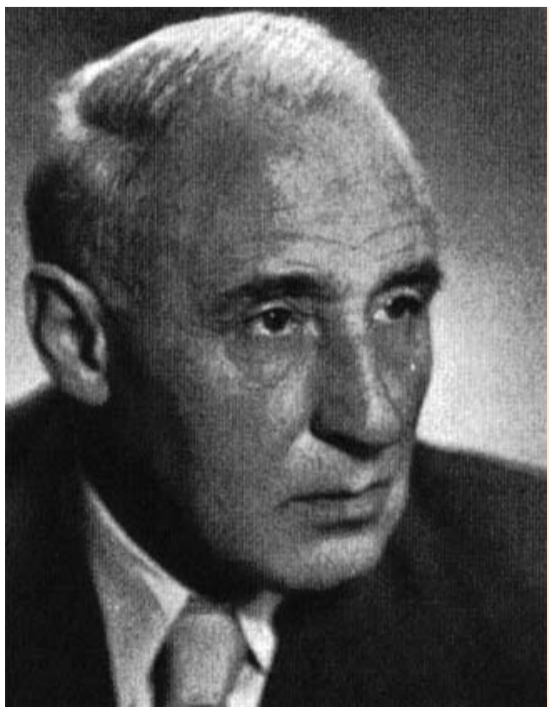

Abb. 2 Hans-Theodor Schreus 1892-1970.

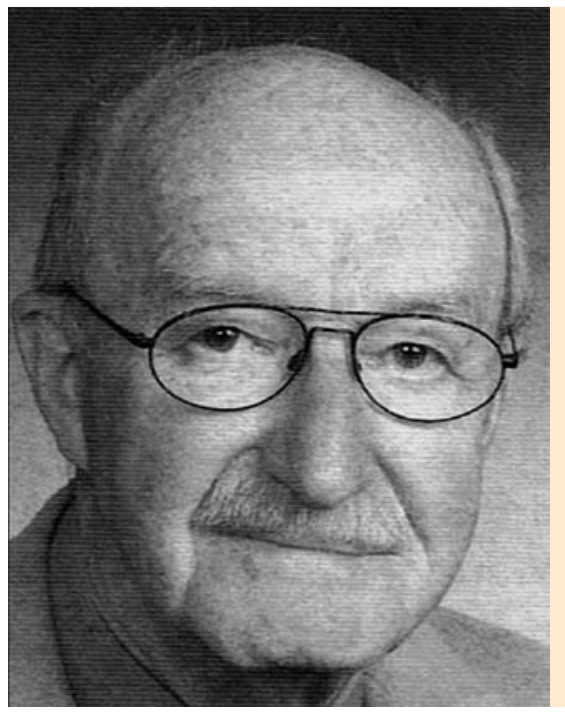

Abb. 3 Erich Landes. seiner Präsidentschaft in der von ihm 1955 gegründeten „Deutschen Gesellschaft für Aesthetische Medizin und ihre Grenzgebiete" zusammenführte. 1960 folgte unter seiner Ägide die Herausgabe eines Publikationsorgans der Gesellschaft, der Zeitschrift „Aesthetische Medizin“.

Waren die Träger der operativen Tradition nach 1890 geboren, so kommt die bis in die Gegenwart wirkende Gründergeneration der operativen Dermatologie in Deutschland aus den 20er-Jahren des 20. Jahrhunderts.

Heinz Egon Kleine-Natrop: geboren 1917

Erich Landes: geboren 1921

Hugo-Constantin Friederich: geboren 1922

Helmut Tritsch: geboren 1923

Erich Landes leitete die Städtische Hautklinik Darmstadt von 1964 bis 1986 ( Abb. 3).

Er betonte die für die dermatologische Therapie notwendige Integration operativer Maßnahmen, der Proktologie und Phlebologie in den klinischen Alltag und gab diese Intention vielen Schülern in seiner menschlich überzeugenden Art und Weise weiter. Er war Gründungsmitglied der „VOD“ und gab 1987, gemeinsam mit Roland Kaufmann, das erste umfangreiche Buch zum Thema „Dermatologische Operationen“ heraus, das in den letzten Jahren in einer überarbeiteten Neuauflage erschien.

Hugo-Constantin Friederich hat 24 Jahre an der UniversitätsHautklinik Tübingen gearbeitet, wo er sich in der dort von Siegmund Limberger geleiteten operativen Abteilung das Handwerkszeug für seine dermatochirurgische Tätigkeit erwarb (๑ Abb. 4).
Von 1970 bis 1991 leitete er die Universitäts-Hautklinik Marburg. In seinen vielseitigen Publikationen setzte er sich nachhaltig dafür ein, dass die operative Therapie ein integraler Bestandteil der klinischen Arbeit jedes Dermatologen sein sollte. 1977 gehörte er zu den Gründungsmitgliedern der VOD. Es muss hervorgehoben werden, dass sein auf vielen Ebenen eingebrachtes Engagement entscheidend zur Durchsetzung und Ausweitung der operativen Dermatologie beigetragen hat, ein Motiv für seine Auszeichnung als Ehrenmitglied der VOD 1992. Obwohl H.-C. Friedrich zu den Gründungsvätern gerechnet werden muss, soll seine große Fairness hervorgehoben werden, dass er in allen seinen Veröffentlichungen die von H. E. Kleine-Natrop 1958 verteidigte und begründete Definition des Begriffs „operative Therapie des Dermatologen“ immer zitiert und als treffend gekennzeichnet hat. Da seine Auffassung von der Dermatologie eng mit dem Bild des Faches von H.-Th. Schreus korrelierte, wurde er 1955 zum ersten Schriftführer der „Deutschen Gesellschaft für Aesthetische Medizin“ gewählt.

Die operative Therapie der Universitäts-Hautklinik Köln ist für zwei Jahrzehnte mit dem Namen von Helmut Tritsch verbunden, der nach Assistenzjahren mit operativer Ausbildung in Heidelberg, Helsinki und Stockholm die Kölner operative Abteilung in der von G. K. Steigleder geleiteten Klinik von 1965 bis 1986 geführt hat $($ Abb. 5)

Er war wegen seiner besonderen Verdienste von 1977 bis 1985 erster und mehrfach wiedergewählter Präsident der VOD.
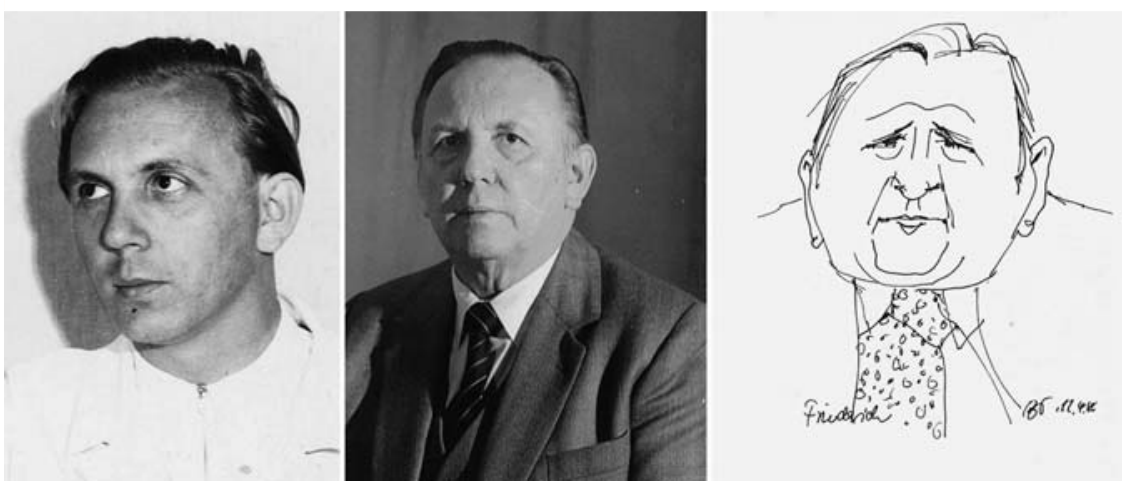

Abb. 4 Hugo-Constantin Friederich, Porträts 1948 bis 1986. 


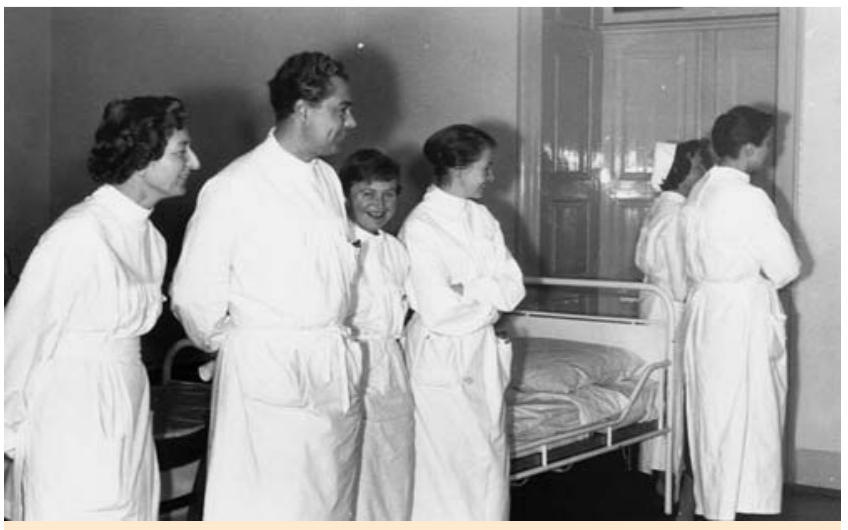

Abb. 5 Helmut Tritsch bei der Visite, Köln.

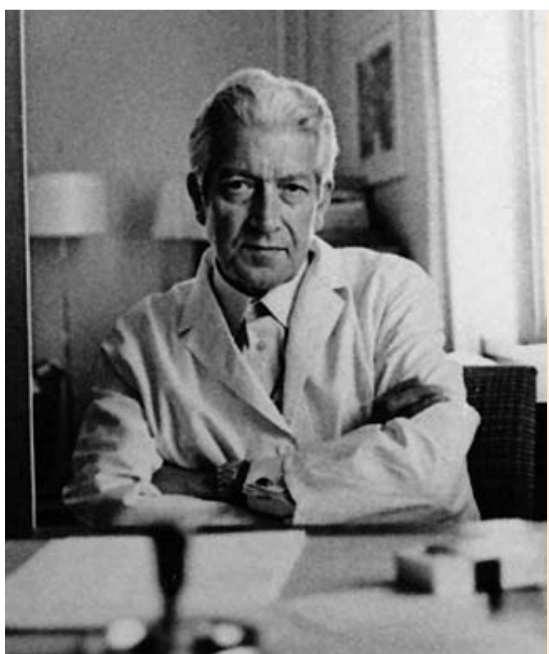

Abb. 6 Heinz Egon Kleine-Natrop, 1917 1986.

Heinz Egon Kleine-Natrop ist sowohl eine Brückenpersönlichkeit zwischen Ost und West als auch die zentrale Persönlichkeit für die Gründung der Dermatochirurgie in der DDR.

\section{Entwicklung der Dermatochirurgie in der DDR $\nabla$}

Die von Günther Sebastian im Jubiläumsband der VOD geschilderte Geschichte der operativen Dermatologie in der DDR soll hier zusammengefasst werden [2].

Aus den ersten Jahren nach dem Ende des 2. Weltkrieges gibt es vereinzelte Berichte zu operativen Aktivitäten aus den Hautkliniken in Chemnitz, Halle und Weimar. In den beiden Jahrzehnten nach 1960 setzte sich die operative Dermatologie weiter durch, in erster Linie in den Universitäts-Hautkliniken von Berlin, Erfurt, Halle, Jena, Leipzig, Magdeburg und Rostock. Die die Entwicklung insgesamt überblickende Einschätzung begründet die Einordnung von Dresden als Zentrum der Dermatochirurgie in der DDR. H. E. Kleine-Natrop hatte Medizin und Zahnmedizin studiert, während seiner Zeit in Kiel operative Tätigkeit praktiziert und bei dem plastischen Chirurgen G. Sanvenero-Rosselli in Mai-

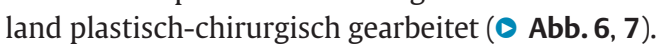

Auf der 7. Weimarer Therapietagung 1961 begründete er die Integration operativen Arbeitens am Organ Haut und definierte für dieses Arbeiten den Begriff „operative Tätigkeit des Dermatologen“, der jede Konkurrenz zu den Chirurgen ausschloss. H. E. Kleine-Natrop veröffentlichte mit seinen Schülern viele Arbeiten zu dem Thema und stellte sowohl das 16. als auch das

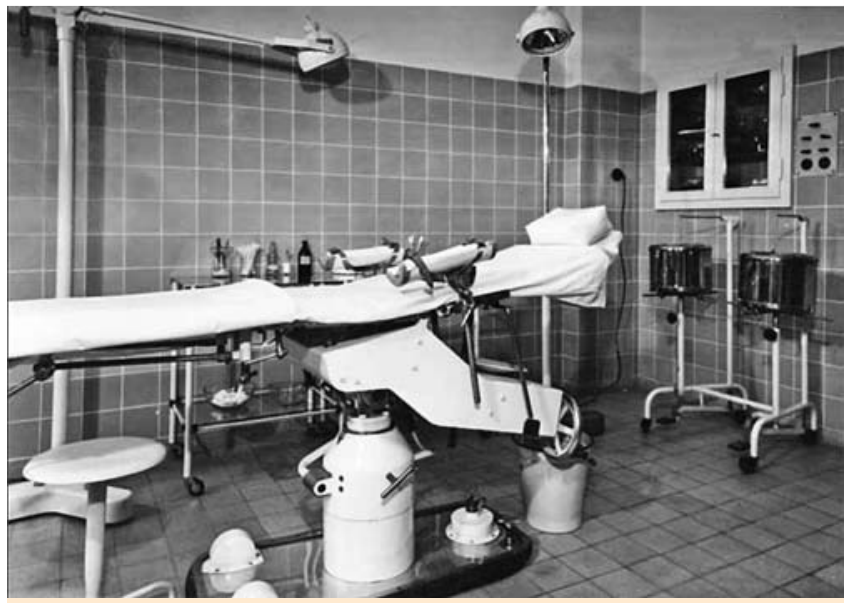

Abb. 7 Operationssaal der Hautklinik Dresden 1959.

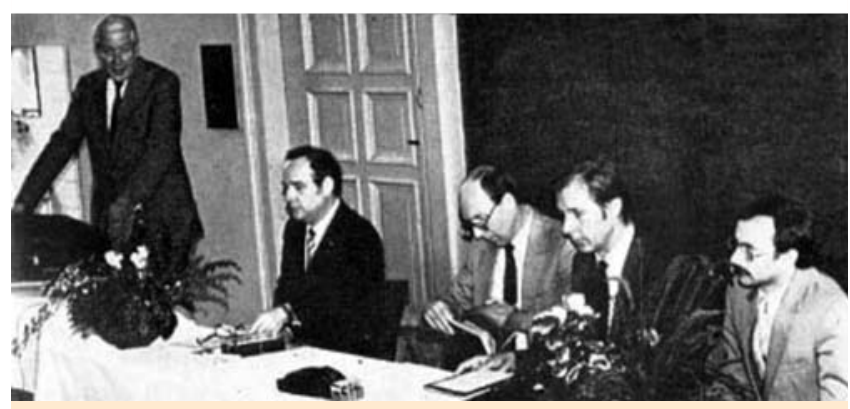

Abb. 8 21. 11. 1982 Berlin: Gründung der Sektion Dermatochirurgie der Gesellschaft für Dermatologie der DDR.

21. Dresdner Dermatologische Gespräch in den Jahren 1976 und 1981 unter Leitthemen aus der Dermatochirurgie. Aus Anlass des 21. Dresdner Dermatologischen Gesprächs gab es am 20.3.1981 ausführliche Gespräche und Diskussionen über die von vielen Dermatologen in der DDR erhobene Forderung der Gründung einer eigenen Fachorganisation für die Inhalte der operativen Tätigkeit an den Hautkliniken. Am 21.11. 1982 erfolgte in Berlin die Gründung der „Sektion Dermatochirurgie der Gesellschaft für Dermatologie der DDR“ ( $\bullet$ Abb. 8).

Die Probleme, die Bedeutung dieser Arbeit im eigenen Fach durchzusetzen und sich in vernünftiger Weise gegenüber anderen Fachrichtungen abzugrenzen, kommt in Kleine-Natrops Formulierung auf der Gründungsveranstaltung 1982 zum Ausdruck: „Es galt, sich nicht nur mit Chirurgen zu vereinigen, sondern auch innerhalb der Dermatologie mussten Tendenzen überwunden werden, die nur in der Anwendung einer nicht fachspezifischen Laborchemie die Zukunft sahen“. Die Sektion fand einen breiten Zuspruch, da die von ihr durchgeführten Veranstaltungen immer von großem Praxisbezug bestimmt waren. Günther Sebastian, Schüler von H. E. Kleine-Natrop, war mit seinen ideenreichen Aktivitäten der Motor der Arbeit, die von Helmut Winter aus der Berliner Charité-Hautklinik immer unterstützt wurde. In den Jahren von 1982 bis 1989 fanden in Dresden und Berlin 8 Arbeitstagungen mit entsprechenden Vorträgen zu Schwerpunktthemen statt ( $\bullet$ Abb. 9). 1988 konnte G. Sebastian in Wilthen/bei Bautzen in der Lausitz ein erstes „Dermatochirurgisches Symposium“ mit internationaler Beteiligung organisieren. Die Teilnahme von H.-C. FriederichMarburg, J. Petres-Kassel und P. Miescher aus Wels in Österreich war für alle Teilnehmer ein bereicherndes Erlebnis und eine große Freude ( $\bullet$ Abb. 10). 


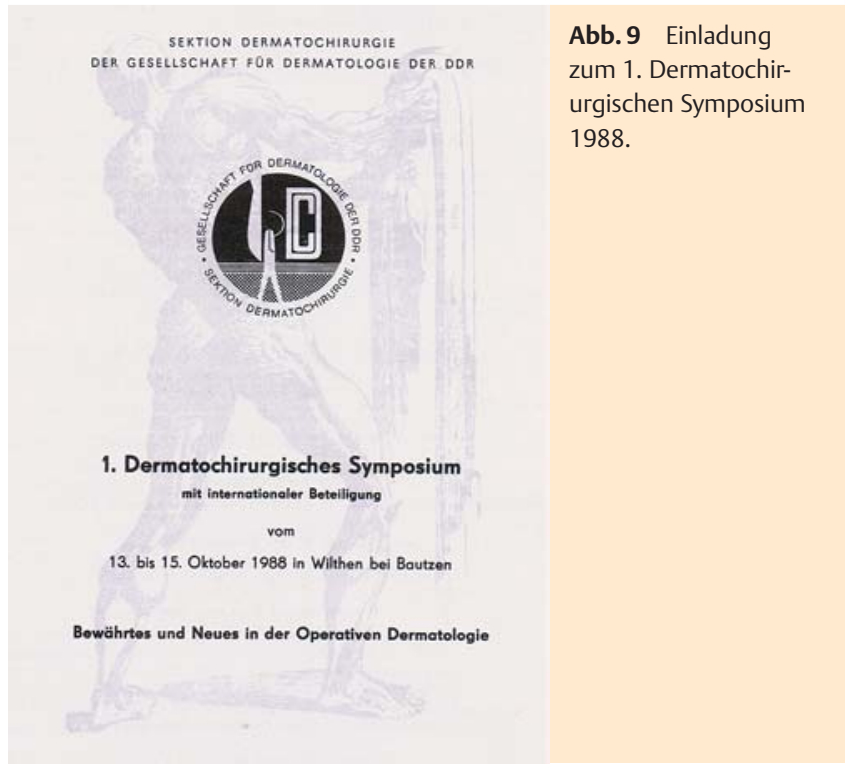

Die beiden in Dresden organisierten, auf praktische Arbeit ausgerichteten Operationskurse fanden großen Zuspruch aus der gesamten DDR und förderten die Ausbreitung einer qualitätsvollen Dermatochirurgie $(\bullet$ Abb. 11).

1990 löste sich nach der deutschen Wiedervereinigung die „Sektion Dermatochirurgie“ auf. Ein Teil der Mitglieder schloss sich der VOD an.

Im Rahmen der Gründungsvorbereitungen der Sektion Dermatochirurgie 1981 in Dresden formulierte H. E. Kleine-Natrop die bis heute geltende Maxime: „Die Signale für die operative Therapie des Dermatologen sind heute mehr denn je nicht auf Konfrontation, sondern auf Kooperation zu stellen.“

\section{Abstract}

\section{Renaissance of Surgical Dermatology after World War II. Development in the GDR \\ $\nabla$}

Biographies and activities of initiators for surgical dermatology in the three decades after the end of World War II are described. In the GDR a „Section for surgical dermatology“ was founded in 1982. Heinz Egon Kleine-Natrop, head of the department of dermatology in Dresden was the centre of all activities on this way. Meetings of this section and practical courses for surgical training are characterised.

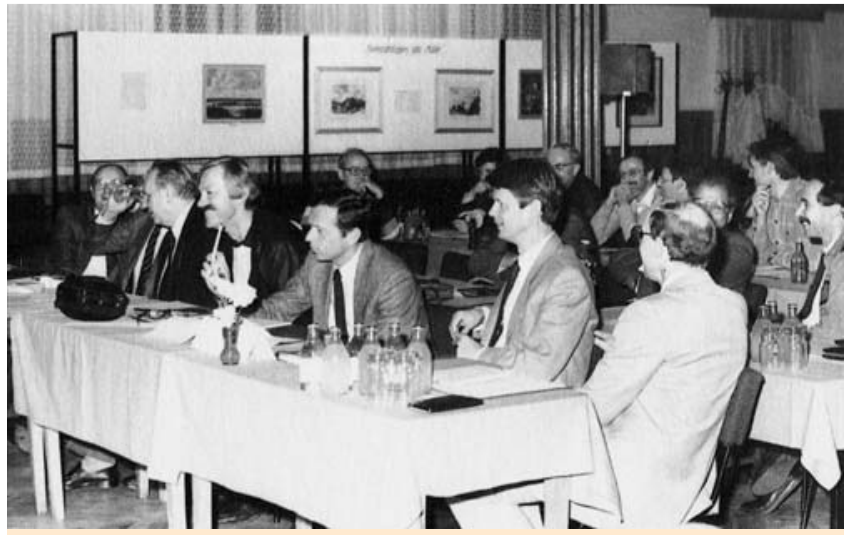

Abb. 10 Dermatochirurgisches Symposium Wilthen bei Bautzen 1988.

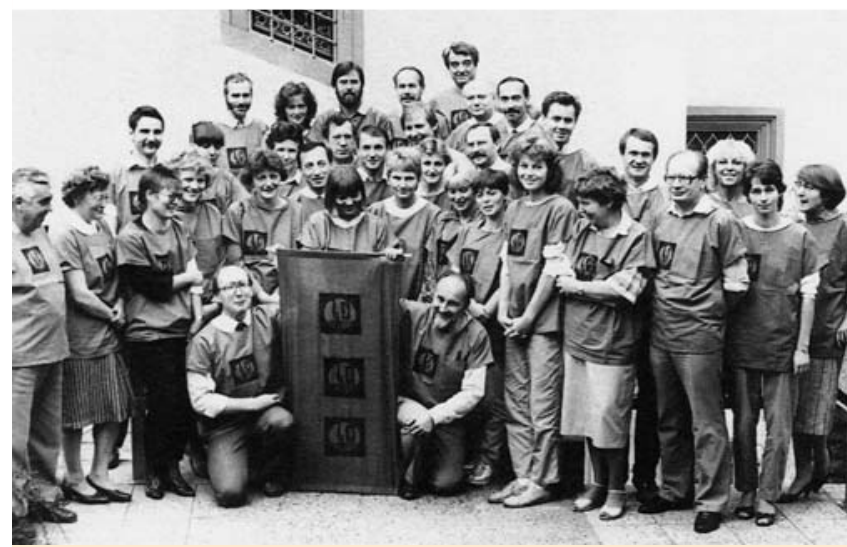

Abb. 11 Teilnehmer des 2. Operationskurses - Dresden 1987.

\section{Literatur}

1 Scholz A, Burg G, Geiges M. Operative Dermatologie, Kryotherapie und Phlebologie. In: Scholz A, Holubar K, Burg G (Hrsg). Geschichte der deutschsprachigen Dermatologie. Weinheim: WILEY-BLACKWELL, 2009: $445-449$

2 Sebastian G. Die Geschichte der operativen Dermatologie in der ehemaligen DDR. In: Konz B, Müller RPA (Hrsg). Zur Lage der operativen und onkologischen Dermatologie. Berlin, Wien: Blackwell, 1977: 41 79 